Received by the editors: March 05, 2020; Accepted: May 30, 2020

\title{
ON NEW INTEGRAL INEQUALITIES USING MIXED CONFORMABLE FRACTIONAL INTEGRALS
}

\author{
Barış ÇELİK and Erhan SET \\ Department of Mathematics, Faculty of Science and Arts, Ordu University, Ordu, TURKEY
}

\begin{abstract}
During the past two decades or so, fractional integral operators have been one of the most important tools in the development of inequalities theory. By this means, a lot generalized intergral inequalities involving various the fractional integral operators have been presented in the literature. Very recently, mixed conformable fractional integral operators has been introduced by $\mathrm{T}$. Abdeljawad and with the help of these operators some new integral inequalities are obtained. The main aim of the paper is to establish some new Chebyshev type fractional integral inequalities by using mixed conformable fractional integral operators.
\end{abstract}

\section{Introduction and Preliminaries}

In the present paper, our work is based on a celebrated functional introduced by Chebyshev 4], which is defined by

$$
T(f, g)=\frac{1}{b-a} \int_{a}^{b} f(x) g(x) d x-\left(\frac{1}{b-a} \int_{a}^{b} f(x) d x\right)\left(\frac{1}{b-a} \int_{a}^{b} g(x) d x\right),
$$

where $f$ and $g$ are two integrable functions which are synchronous on $[a, b]$, i.e.

$$
(f(x)-f(y))(g(x)-g(y)) \geq 0
$$

for any $x, y \in[a, b]$, then the Chebyshev inequality is given by $T(f, g) \leq 0$.

2020 Mathematics Subject Classification. Primary 26A33; Secondary 26D10, 26D15.

Keywords and phrases. Chebsyev inequality, mixed conformable fractional integral.

bariscelik15@hotmail.com; erhanset@yahoo.com (Corresponding author)

(D) 0000-0001-5372-7543; 0000-0003-1364-5396. 
The Chebyshev functional (1) has many applications in numerical quadrature, transform theory, probability, study of existence for solutions of differential equations, and in statistical problems. Moreover, under suitable assumptions (Chebyshev inequality, Grüss inequality, Minkowski inequality, Hermite-Hadamard inequality, Ostrowski inequality etc.), inequalities are playing a significant role in the field of mathematical sciences, particularly, in the theory of approximations.

A remarkably large number inequalities of above type involving the special fractional integral (such as the Riemann-Liouville, conformable, Erdélyi-Kober, Katugampola, Hadamard and Weyl types) have been investigated by many researchers and received considerable attention to it (see 8 10,14,16]).

Now, some fractional integral operators and Chebyshev type inequalities obtained with the help of these operators will be given in the following order:

Definition 1. Let $f \in L[a, b]$. The Riemann-Liouville integrals $J_{a+}^{\alpha} f$ and $J_{b-}^{\alpha} f$ of order $\alpha \in R^{+}$with $a \in R_{0}^{+}$are defined, respectively, by

$$
J_{a+}^{\alpha} f(x)=\frac{1}{\Gamma(\alpha)} \int_{a}^{x}(x-t)^{\alpha-1} f(t) d t \quad(x>a)
$$

and

$$
J_{b-}^{\alpha} f(x)=\frac{1}{\Gamma(\alpha)} \int_{x}^{b}(t-x)^{\alpha-1} f(t) d t \quad(x<b)
$$

where $\Gamma$ is the familiar Gamma function (see, e.g., [19, Section 1.1]). It is noted that $J_{a+}^{1} f(x)$ and $J_{b-}^{1} f(x)$ become the usual Riemann integrals.

Theorem 2. [7] Let $p$ be a positive function on $[0, \infty[$ and let $f$ and $g$ be two differentiable functions on $\left[0, \infty\left[\right.\right.$. If $f^{\prime} \in L_{r}\left(\left[0, \infty[), g^{\prime} \in L_{s}\left(\left[0, \infty[), r^{-1}+s^{-1}=1\right.\right.\right.\right.$, then for all $t>0, \alpha>0$, we have

$$
\begin{aligned}
& 2\left|J^{\alpha} p(t) J^{\alpha} p f g(t)-J^{\alpha} p f(t) J^{\alpha} p g(t)\right| \\
\leq & \frac{\left\|f^{\prime}\right\|_{r}\left\|g^{\prime}\right\|_{s}}{\Gamma^{2}(\alpha)} \int_{0}^{t} \int_{0}^{t}(t-\tau)^{\alpha-1}(t-\rho)^{\alpha-1}|\tau-\rho| p(\tau) p(\rho) d \tau d \rho \\
\leq & \left\|f^{\prime}\right\|_{r}\left\|g^{\prime}\right\|_{s} t\left(J^{\alpha} p(t)\right)^{2} .
\end{aligned}
$$

Theorem 3. [7] Let $p$ be a positive function on $[0, \infty[$ and let $f$ and $g$ be two differentiable functions on $\left[0, \infty\left[\right.\right.$. If $f^{\prime} \in L_{r}\left(\left[0, \infty[), g^{\prime} \in L_{s}\left(\left[0, \infty[), r^{-1}+s^{-1}=1\right.\right.\right.\right.$, then for all $t>0, \alpha, \beta>0$, we have

$$
\begin{aligned}
& \left|J^{\alpha} p(t) J^{\beta} p f g(t)+J^{\beta} p(t) J^{\alpha} p f g(t)-J^{\alpha} p f(t) J^{\beta} p g(t)-J^{\beta} p f(t) J^{\alpha} p g(t)\right| \\
\leq & \frac{\left\|f^{\prime}\right\|_{r}\left\|g^{\prime}\right\|_{s}}{\Gamma(\alpha) \Gamma(\beta)} \int_{0}^{t} \int_{0}^{t}(t-\tau)^{\alpha-1}(t-\rho)^{\alpha-1}|\tau-\rho| p(\tau) p(\rho) d \tau d \rho \\
\leq & \left\|f^{\prime}\right\|_{r}\left\|g^{\prime}\right\|_{s} t J^{\alpha} p(t) J^{\beta} p(t) .
\end{aligned}
$$

Definition 4. Let $\alpha>0, \mu>-1, \beta, \eta \in R$; then, a generalized fractional integral $I_{t}^{\alpha, \beta, \eta, \mu}$ (in terms of the Gauss hypergeometric function) of order $\alpha$ for a real-valued 
continuous function $f(t)$ is defined by [5] (see also [12])

$$
I_{t}^{\alpha, \beta, \eta, \mu}\{f(t)\}=\frac{t^{-\alpha-\beta-2 \mu}}{\Gamma(\alpha)} \int_{0}^{t} \tau^{\mu}(t-\tau)^{\alpha-1}{ }_{2} F_{1}\left(\alpha+\beta+\mu-\eta ; \alpha ; 1-\frac{\tau}{t}\right) f(\tau) d \tau,
$$

where the function ${ }_{2} F_{1}(-)$ appearing as a kernel for the operator (2) is the Gaussian hypergeometric function defined by

$$
{ }_{2} F_{1}(a, b ; c ; t)=\sum_{n=0}^{\infty} \frac{(a) n(b) n}{(c)_{n}} \frac{t^{n}}{n !},
$$

and $(a)_{n}$ is the Pochhammer symbol

$$
(a)_{n}=a(a+1) \ldots(a+n-1), \quad(a)_{0}=1 .
$$

Theorem 5. [2] Let $p$ be a positive function and let $f$ and $g$ be two synchronous functions on $[0, \infty)$. If $f^{\prime} \in L_{r}([0, \infty)), g^{\prime} \in L_{s}([0, \infty)), r^{-1}+s^{-1}=1$, then (for all $t>0, \beta<1, \mu>-1, \alpha>\max \{0,-\beta-\mu\}, \beta-1<\eta<0)$

$$
\begin{aligned}
& 2\left|I_{t}^{\alpha, \beta, \eta, \mu}\{p(t)\} I_{t}^{\alpha, \beta, \eta, \mu}\{p(t) f(t) g(t)\}-I_{t}^{\alpha, \beta, \eta, \mu}\{p(t) f(t)\} I_{t}^{\alpha, \beta, \eta, \mu}\{p(t) g(t)\}\right| \\
\leq & \frac{t^{-2 \alpha-2 \beta-4 \mu}\left\|f^{\prime}\right\|\left\|_{r}\right\| g^{\prime} \|_{s}}{\Gamma^{2}(\alpha)} \int_{0}^{t} \int_{0}^{t} \tau^{\mu} \rho^{\mu}(t-\tau)^{\alpha-1}(t-\rho)^{\alpha-1} \\
& \times{ }_{2} F_{1}\left(\alpha+\beta+\mu,-\eta ; \alpha ; 1-\frac{\tau}{t}\right)_{2} F_{1}\left(\alpha+\beta+\mu,-\eta ; \alpha ; 1-\frac{\rho}{t}\right) p(\tau) p(\rho)|\tau-\rho| d \tau d \rho \\
\leq & \left\|f^{\prime}\right\|_{r}\left\|g^{\prime}\right\|_{s} t\left(I_{t}^{\alpha, \beta, \eta, \mu}\{p(t)\}\right)^{2} .
\end{aligned}
$$

Theorem 6. [2] Let $p$ be a positive function and let $f$ and $g$ be two synchronous functions on $[0, \infty)$. If $f^{\prime} \in L_{r}([0, \infty)), g^{\prime} \in L_{s}([0, \infty)), r>1 r^{-1}+s^{-1}=1$, then

$$
\begin{aligned}
& \mid I_{t}^{\alpha, \beta, \eta, \mu}\{p(t)\} I_{t}^{\gamma, \delta, \zeta, \nu}\{p(t) f(t) g(t)\}+I_{t}^{\gamma, \delta, \zeta, \nu}\{p(t)\} I_{t}^{\alpha, \beta, \eta, \mu}\{p(t) f(t) g(t)\} \\
& -I_{t}^{\alpha, \beta, \eta, \mu}\{p(t) f(t)\} I_{t}^{\gamma, \delta, \zeta, \nu}\{p(t) g(t)\}-I_{t}^{\gamma, \delta, \zeta, \nu}\{p(t) f(t)\} I_{t}^{\alpha, \beta, \eta, \mu}\{p(t) g(t)\} \mid \\
\leq & \frac{t^{-\alpha-\beta-\gamma-\delta-2(\mu+\nu)}\left\|f^{\prime}\right\|_{r}\left\|g^{\prime}\right\|_{s}}{\Gamma(\alpha) \Gamma(\gamma)} \int_{0}^{t} \int_{0}^{t} \tau^{\mu} \rho^{\mu}(t-\tau)^{\alpha-1}(t-\rho)^{\gamma-1} \\
& \times{ }_{2} F_{1}\left(\alpha+\beta+\mu,-\eta ; \alpha ; 1-\frac{\tau}{t}\right){ }_{2} F_{1}\left(\gamma+\delta+\nu,-\zeta ; \gamma ; 1-\frac{\rho}{t}\right) p(\tau) p(\rho)|\tau-\rho| d \tau d \rho \\
\leq & \left\|f^{\prime}\right\|_{r}\left\|g^{\prime}\right\|_{s} t I_{t}^{\gamma, \delta, \zeta, \nu}\{p(t)\}, I_{t}^{\alpha, \beta, \eta, \mu}\{p(t)\},
\end{aligned}
$$

for all $t>0, \alpha>\max \{0,-\beta-\mu\}, \beta<1, \mu>-1, \beta-1<\eta<0, \gamma>$ $\max \{0,-\delta-\nu\}, \delta<1, \nu>-1, \delta-1<\zeta<0$.

Definition 7. [11] The Hadamard fractional integral of order $\alpha \in R^{+}$of a function $f(t)$, for all $t>1$, is defined as

$$
{ }_{H} J^{\alpha}\{f(t)\}=\frac{1}{\Gamma(\alpha)} \int_{1}^{t}\left(\log \frac{t}{\tau}\right)^{\alpha-1} f(\tau) \frac{d \tau}{\tau} .
$$


Theorem 8. [13] Let $p$ be a positive function and let $f$ and $g$ be two differentiable functions on $[1, \infty)$. If $f^{\prime} \in L_{r}([1, \infty)), g^{\prime} \in L_{s}([1, \infty)), r>1, r^{-1}+s^{-1}=1$, then for all $t>1$ and $\alpha>0$,

$$
\begin{aligned}
& \left.2\right|_{H} J^{\alpha}\{p(t)\}_{H} J^{\alpha}\{p(t) f(t) g(t)\}-{ }_{H} J^{\alpha}\{p(t) f(t)\}_{H} J^{\alpha}\{p(t) g(t)\} \mid \\
\leq & \frac{\left\|f^{\prime}\right\|_{r}\left\|g^{\prime}\right\|_{s}}{\Gamma^{2}(\alpha)} \int_{1}^{t} \int_{1}^{t}\left(\log \frac{t}{\tau}\right)^{\alpha-1}\left(\log \frac{t}{\rho}\right)^{\alpha-1} \frac{p(\tau) p(\rho)}{\tau \rho}|\tau-\rho| d \tau d \rho \\
\leq & \left\|f^{\prime}\right\|_{r}\left\|g^{\prime}\right\|_{s} t\left({ }_{H} J^{\alpha}\{p(t)\}\right)^{2} .
\end{aligned}
$$

Theorem 9. [13] Let $p$ be a positive function and let $f$ and $g$ be two differentiable functions on $[1, \infty)$. If $f^{\prime} \in L_{r}([1, \infty)), g^{\prime} \in L_{s}([1, \infty)), r>1, r^{-1}+s^{-1}=1$, then

$$
\begin{aligned}
& \mid{ }_{H} J^{\alpha}\{p(t)\}_{H} J^{\beta}\{p(t) f(t) g(t)\}+_{H} J^{\beta}\{p(t)\}_{H} J^{\alpha}\{p(t) f(t) g(t)\} \\
& -{ }_{H} J^{\alpha}\{p(t) f(t)\}_{H} J^{\beta}\{p(t) g(t)\}-_{H} J^{\beta}\{p(t) f(t)\}_{H} J^{\alpha}\{p(t) g(t)\} \mid \\
\leq & \frac{\left\|f^{\prime}\right\|_{r}\left\|g^{\prime}\right\|_{s}}{\Gamma(\alpha) \Gamma(\beta)} \int_{1}^{t} \int_{1}^{t}\left(\log \frac{t}{\tau}\right)^{\alpha-1}\left(\log \frac{t}{\rho}\right)^{\alpha-1} \frac{p(\tau) p(\rho)}{\tau \rho}|\tau-\rho| d \tau d \rho \\
\leq & \left\|f^{\prime}\right\|_{r}\left\|g^{\prime}\right\|_{s} t_{H} J^{\alpha}\{p(t)\}_{H} J^{\beta}\{p(t)\},
\end{aligned}
$$

for all $t>1, \alpha>0$ and $\beta>0$.

Definition 10. [12] Let $\alpha>0, \beta>0$ and $\eta \in R$, then the Erdélyi-Kober fractional integral operators $I_{\beta}^{\eta}, \alpha$ of order $\alpha$ for a real-valued continuous function $f(t)$ is defined as

$$
\begin{aligned}
I_{\beta}^{\eta}, \alpha\{f(t)\} & =\frac{t^{-\beta(\eta+\alpha)}}{\Gamma(\alpha)} \int_{0}^{t} \tau^{\beta \eta}\left(t^{\beta}-\tau^{\beta}\right)^{\alpha-1} f(\tau) d\left(\tau^{\beta}\right) \\
& =\frac{\beta t^{-\beta(\eta+\alpha)}}{\Gamma(\alpha)} \int_{0}^{t} \tau^{\beta(\eta+1)-1}\left(t^{\beta}-\tau^{\beta}\right)^{\alpha-1} f(\tau) d \tau .
\end{aligned}
$$

Theorem 11. 2] Suppose that $p$ be a positive function, $f$ and $g$ be differentiable functions on $[0, \infty), f^{\prime} \in L_{r}([0, \infty)), g^{\prime} \in L_{s}([0, \infty))$ such that $r^{-1}+s^{-1}=1$ with $r>1$. Then for all $t>0, \alpha>0, \beta>0, \eta \in R$ and $\eta>-1$ :

$$
\begin{aligned}
& 2\left|I_{\beta}^{\eta, \alpha}\{p(t)\} I_{\beta}^{\eta, \alpha}\{p(t) f(t) g(t)\}-I_{\beta}^{\eta, \alpha}\{p(t) f(t)\} I_{\beta}^{\eta, \alpha}\{p(t) g(t)\}\right| \\
\leq & \frac{\beta^{2} t^{-2 \beta \eta+\alpha}\left\|f^{\prime}\right\|_{r}\left\|g^{\prime}\right\|_{s}}{\Gamma^{2}(\alpha)} \int_{0}^{t} \int_{0}^{t} \tau^{\mu} \rho^{\mu}(t-\tau)^{\alpha-1}(t-\rho)^{\alpha-1} \\
& \times{ }_{2} F_{1}\left(\alpha+\beta+\mu,-\eta ; \alpha ; 1-\frac{\tau}{t}\right)_{2} F_{1}\left(\alpha+\beta+\mu,-\eta ; \alpha ; 1-\frac{\rho}{t}\right) p(\tau) p(\rho)|\tau-\rho| d \tau d \rho \\
\leq & \left\|f^{\prime}\right\|_{r}\left\|g^{\prime}\right\|_{s} t\left(I_{\beta}^{\eta, \alpha}\{p(t)\}\right)^{2} .
\end{aligned}
$$

Theorem 12. [2] Suppose that $p$ be a positive function, $f$ and $g$ be differentiable functions on $[0, \infty), f^{\prime} \in L_{r}([0, \infty)), g^{\prime} \in L_{s}([0, \infty))$ such that $r>1$ and $r^{-1}+$ 
$s^{-1}=1$. Then for all $t>0$ the following inequality holds:

$$
\begin{aligned}
& \mid I_{\beta}^{\eta, \alpha}\{p(t)\} I_{\delta}^{\zeta, \gamma}\{p(t) f(t) g(t)\}+I_{\delta}^{\zeta, \gamma}\{p(t)\} I_{\beta}^{\eta, \alpha}\{p(t) f(t) g(t)\} \\
& -I_{\beta}^{\eta, \alpha}\{p(t) f(t)\} I_{\delta}^{\zeta, \gamma}\{p(t) g(t)\}-I_{\delta}^{\zeta, \gamma}\{p(t) f(t)\} I_{\beta}^{\eta, \alpha}\{p(t) g(t)\} \mid \\
\leq & \frac{\beta \delta t^{-\beta(\eta+\alpha)-\delta(\zeta+\gamma)}\left\|f^{\prime}\right\|_{r}\left\|g^{\prime}\right\|_{s}}{\Gamma(\alpha) \Gamma(\gamma)} \int_{0}^{t} \int_{0}^{t} \tau^{\beta(\eta+1)-1} \rho^{\delta(\zeta+1)-1}\left(t^{\beta}-\tau^{\beta}\right)^{\alpha-1}\left(t^{\delta}-\rho^{\delta}\right)^{\gamma-1} \\
& \times p(\tau) p(\rho)|\tau-\rho| d \tau d \rho \\
\leq & \left\|f^{\prime}\right\|_{r}\left\|g^{\prime}\right\|_{s} t I_{\beta}^{\eta, \alpha}\{p(t)\}, I_{\delta}^{\zeta, \gamma}\{p(t)\},
\end{aligned}
$$

where $\alpha, \beta, \gamma, \delta>0, \eta, \zeta \in R$ and $\eta>-1$ and $\zeta>-1$.

Definition 13. 15$]$ Let $p \geq 0, q>0, \omega, \delta, \lambda, \sigma, c, \rho \in C, \Re(c)>0, \Re(\rho)>0$ and $\Re(\sigma)>0$. Let $f \in L[a, b]$ and $x \in[a, b]$. Then the fractional integral operator $\left(\epsilon_{a^{+}, \rho, \sigma}^{\omega, \delta, q} f\right)$ defined by Rahman et al. is as the following:

$$
\left(\epsilon_{a^{+}, \rho, \sigma}^{\omega, \delta, q} f\right)(x)=\int_{a}^{x}(x-\tau)^{\sigma-1} E_{p, \sigma}^{\delta, q, c}\left(\omega(x-\tau)^{\rho} ; p\right) f(\tau) d \tau
$$

where

$$
E_{\rho, \sigma}^{\delta, q, c}(z ; p)=\sum_{n=0}^{\infty} \frac{B_{p}(\delta+n q, c-\delta)}{B(\delta, c-\delta)} \frac{(c)_{n q}}{\Gamma(\rho n+\sigma)} \frac{z^{n}}{n !}
$$

and $B_{p}(x, y)$ is an extension of Beta function defined in [15]

$$
B_{p}(x, y)=\int_{0}^{1} t^{x-1}(1-t)^{y-1} e^{-\frac{p}{t(1-t)}} d t \quad x, y, p>0
$$

where $\Re(p)>0, \Re(x)>0$ and $\Re(y)>0$. Also, here $B$ is familiar Beta function as follows:

$$
B(a, b)=\frac{\Gamma(a) \Gamma(b)}{\Gamma(a+b)}=\int_{0}^{1} t^{a-1}(1-t)^{b-1} d t, \quad a, b>0 .
$$

Theorem 14. [17] Let $t$ be a positive function on $[0, \infty)$ and let $f$ and $g$ be two differentiable functions on $[0, \infty)$. If $f^{\prime} \in L_{r}([0, \infty)), g^{\prime} \in L_{s}([0, \infty)), r^{-1}+s^{-1}=$ 1 , then for all $x>0, \alpha, \beta>0$, we have

$$
\begin{aligned}
& 2\left|\left(\epsilon_{0^{+}, \alpha, \beta, \sigma}^{\omega, \delta, q, r} t f g\right)(x ; p)\left(\epsilon_{0^{+}, \alpha, \beta, \sigma}^{\omega, \delta, q, c} t\right)(x ; p)-\left(\epsilon_{0^{+}, \alpha, \beta, \sigma}^{\omega, \delta, q, r, c} t f\right)(x ; p)\left(\epsilon_{0^{+}, \alpha, \beta, \sigma}^{\omega, \delta, q, r, c} t g\right)(x ; p)\right| \\
\leq & \left\|f^{\prime}\right\|_{r}\left\|g^{\prime}\right\|_{s} \int_{0}^{x} \int_{0}^{x}(x-\tau)^{\beta-1}(x-\rho)^{\beta-1}|\tau-\rho| t(\tau) t(\rho) \\
& \times E_{0^{+}, \alpha, \beta, \sigma}^{\omega, \delta, q, r, c}\left(\omega(x-\tau)^{\alpha} ; p\right) E_{0^{+}, \alpha, \beta, \sigma, \sigma}^{\omega, \delta, q, c}\left(\omega(x-\rho)^{\alpha} ; p\right) d \tau d \rho \\
\leq & \left\|f^{\prime}\right\|_{r}\left\|g^{\prime}\right\|_{s} x\left(\left(\epsilon_{0^{+}, \alpha, \beta, \sigma}^{\omega, \delta, q, c} t\right)(x ; p)\right)^{2} .
\end{aligned}
$$


Theorem 15. [17] Let $t$ be a positive function on $[0, \infty)$ and let $f$ and $g$ be two differentiable functions on $[0, \infty)$. If $f^{\prime} \in L_{r}([0, \infty)), g^{\prime} \in L_{s}([0, \infty)), r^{-1}+s^{-1}=$ 1 , then for all $x>0, \alpha, \beta, \lambda, \theta>0$, we have

$$
\begin{aligned}
& \mid\left(\epsilon_{0^{+}, \alpha, \beta, \sigma}^{\omega, \delta, q, c} t\right)(x ; p)\left(\epsilon_{0^{+}, \lambda, \theta, p}^{\omega, q, r, c} t f g\right)(x ; p)+\left(\epsilon_{0^{+}, \lambda, \theta, p}^{\omega, \delta, q, r, c} t\right)(x ; p)\left(\epsilon_{0^{+}, \alpha, \beta, \sigma}^{\omega, \delta, q, r, c} t f g\right)(x ; p) \\
& -\left(\epsilon_{0^{+}, \alpha, \beta, \beta, \sigma}^{\omega, \delta, r, c} t f\right)(x ; p)\left(\epsilon_{0^{+}, \lambda, \theta, p}^{\omega, \delta, q, r, c} t g\right)(x ; p)-\left(\epsilon_{0^{+}, \lambda, \theta, p}^{\omega, \delta, r, c} t f\right)(x ; p)\left(\epsilon_{0^{+}, \alpha, \beta, \sigma}^{\omega, \delta, r, c} t g\right)(x ; p) \mid \\
\leq & \left\|f^{\prime}\right\|_{r}\left\|g^{\prime}\right\|_{s} \int_{0}^{x} \int_{0}^{x}(x-\tau)^{\beta-1}(x-\rho)^{\theta-1}|\tau-\rho| t(\tau) t(\rho) \\
& \times E_{0^{+}, \alpha, \beta, \sigma}^{\omega, \delta, q, r}\left(\omega(x-\tau)^{\alpha} ; p\right) E_{0^{+}, \lambda, \theta, p}^{\omega, \delta, q, r, c}\left(\omega(x-\rho)^{\lambda} ; p\right) d \tau d \rho \\
\leq & \left\|f^{\prime}\right\|_{r}\left\|g^{\prime}\right\|_{s} x\left(\epsilon_{0^{+}, \alpha, \beta, \sigma}^{\omega, \delta, q, r, c} t\right)(x ; p)\left(\epsilon_{0^{+}, \lambda, \theta, p}^{\omega, \delta, q, c} t\right)(x ; p) .
\end{aligned}
$$

Definition 16. [1] Let $f$ be defined on $[a, b]$ and $\alpha \in C, \operatorname{Re}(\alpha)>0, \rho>0$. Then

(i) The mixed left conformable fractional integral of $f$ is defined by

$$
{ }_{a}^{b} \mathfrak{J}^{\alpha, \rho} f(x)=\frac{1}{\Gamma(\alpha)} \int_{a}^{x} f(s)\left(\frac{(b-s)^{\rho}-(b-x)^{\rho}}{\rho}\right)^{\alpha-1}(b-s)^{\rho-1} d s .
$$

and

(ii) The mixed right conformable fractional integral of $f$ is defined by

$$
{ }_{\mathfrak{J}_{b}^{\alpha, \rho}}^{\alpha} f(x)=\frac{1}{\Gamma(\alpha)} \int_{x}^{b} f(s)\left(\frac{(s-a)^{\rho}-(x-a)^{\rho}}{\rho}\right)^{\alpha-1}(s-a)^{\rho-1} d s .
$$

For recent results related to this operators, we refer the reader [1,6, 18.

\section{Main Results}

We obtain in this section certain integral inequalities for the differentiable functions involving the mixed conformable fractional integral operator.

Theorem 17. Let $p$ be a positive function on $[0, \infty[$ and let $f$ and $g$ be two differentiable functions on $\left[0, \infty\left[\right.\right.$. If $f^{\prime} \in L_{r}\left(\left[0, \infty[), g^{\prime} \in L_{s}\left(\left[0, \infty[), r^{-1}+s^{-1}=1\right.\right.\right.\right.$, then for all $t>0, \alpha, \rho>0$, we have

$$
\begin{aligned}
& 2\left|{ }_{0}^{b} \mathfrak{J}^{\alpha, \rho} p(t){ }_{0}^{b} \mathfrak{J}^{\alpha, \rho} p f g(t)-{ }_{0}^{b} \mathfrak{J}^{\alpha, \rho} f p(t){ }_{0}^{b} \mathfrak{J}^{\alpha, \rho} p g(t)\right| \\
\leq & \frac{\left\|f^{\prime}\right\|_{r}\left\|g^{\prime}\right\|_{s}}{\Gamma^{2}(\alpha)}\left[\int_{0}^{t} \int_{0}^{t}\left(\frac{(b-x)^{\rho}-(b-t)^{\rho}}{\rho}\right)^{\alpha-1}(b-x)^{\rho-1}\right. \\
& \left.\times\left(\frac{(b-y)^{\rho}-(b-t)^{\rho}}{\rho}\right)^{\alpha-1}(b-y)^{\rho-1}|x-y| p(x) p(y) d x d y\right] \\
\leq & \left\|f^{\prime}\right\|_{r}\left\|g^{\prime}\right\|_{s} t\left({ }_{0}^{b} \mathfrak{J}^{\alpha, \rho} p(t)\right)^{2} .
\end{aligned}
$$


Proof. Let $f$ and $g$ be two functions satisfying the conditions of Theorem 17 and let $p$ be a positive function on $[0, \infty[$.

Define

$$
H(x, y):=(f(x)-f(y)(g(x)-g(y))) ; \quad x, y \in(0, t), t>0 .
$$

Multiplying (6) by $\frac{1}{\Gamma(\alpha)}\left(\frac{(b-x)^{\rho}-(b-t)^{\rho}}{\rho}\right)^{\alpha-1}(b-x)^{\rho-1} p(x)$ and integrating the resulting identity with respect to $x$ from 0 to $t$, we can write

$$
\begin{aligned}
& \frac{1}{\Gamma(\alpha)} \int_{0}^{t}\left(\frac{(b-x)^{\rho}-(b-t)^{\rho}}{\rho}\right)^{\alpha-1}(b-x)^{\rho-1} p(x) H(x, y) d x \\
= & { }_{0}^{b} \mathfrak{J}^{\alpha, \rho} p f g(t)-f(y){ }_{0}^{b} \mathfrak{J}^{\alpha, \rho} p g(t)-g(y){ }_{0}^{b} \mathfrak{J}^{\alpha, \rho} p f(t)+f(y) g(y){ }_{0}^{b} \mathfrak{J}^{\alpha, \rho} p(t) .
\end{aligned}
$$

Now, multiplying (7) by $\frac{1}{\Gamma(\alpha)}\left(\frac{(b-y)^{\rho}-(b-t)^{\rho}}{\rho}\right)^{\alpha-1}(b-y)^{\rho-1} p(y)$ and integrating the resulting identity with respect to $y$ from 0 to $t$, we can write

$$
\begin{aligned}
& \frac{1}{\Gamma(\alpha)} \int_{0}^{t} \int_{0}^{t}\left(\frac{(b-x)^{\rho}-(b-t)^{\rho}}{\rho}\right)^{\alpha-1}(b-x)^{\rho-1} \\
& \times\left(\frac{(b-y)^{\rho}-(b-t)^{\rho}}{\rho}\right)^{\alpha-1}(b-y)^{\rho-1} p(x) p(y) H(x, y) d x \\
= & 2\left({ }_{0}^{b} \mathfrak{J}^{\alpha, \rho} p(t){ }_{0}^{b} \mathfrak{J}^{\alpha, \rho} p f g(t)-{ }_{0}^{b} \mathfrak{J}^{\alpha, \rho} p f(t){ }_{0}^{b} \mathfrak{J}^{\alpha, \rho} p g(t)\right) .
\end{aligned}
$$

On the other hand, we have

$$
H(x, y):=\int_{x}^{y} \int_{x}^{y} f^{\prime}(u) g^{\prime}(w) d u d w .
$$

Using Hölder inequality for double integral, we can write

$$
|H(x, y)| \leq\left.\left.\left.\left.\left|\int_{x}^{y} \int_{x}^{y}\right| f^{\prime}(u)\right|^{r} d u d w\right|^{r^{-1}}\left|\int_{x}^{y} \int_{x}^{y}\right| g^{\prime}(w)\right|^{s} d u d w\right|^{s^{-1}} .
$$

Since

$$
\left.\left.\left|\int_{x}^{y} \int_{x}^{y}\right| f^{\prime}(u)\right|^{r} d u d w\right|^{r^{-1}}=\left.\left.|x-y|^{r^{-1}}\left|\int_{x}^{y}\right| f^{\prime}(u)\right|^{r} d u\right|^{r^{-1}}
$$

and

$$
\left.\left.\left|\int_{x}^{y} \int_{x}^{y}\right| g^{\prime}(w)\right|^{s} d u d w\right|^{s^{-1}}=\left.\left.|x-y|^{s^{-1}}\left|\int_{x}^{y}\right| g^{\prime}(w)\right|^{s} d w\right|^{s^{-1}},
$$

then, we can estimate $H$ as follows:

$$
|H(x, y)| \leq\left.\left.\left.\left.|x-y|\left|\int_{x}^{y}\right| f^{\prime}(u)\right|^{r} d u\right|^{r^{-1}}\left|\int_{x}^{y}\right| g^{\prime}(w)\right|^{s} d u\right|^{s^{-1}} .
$$

On the other hand, we have

$$
\frac{1}{\Gamma^{2}(\alpha)} \int_{0}^{t} \int_{0}^{t}\left(\frac{(b-x)^{\rho}-(b-t)^{\rho}}{\rho}\right)^{\alpha-1}(b-x)^{\rho-1}
$$




$$
\begin{aligned}
& \times\left(\frac{(b-y)^{\rho}-(b-t)^{\rho}}{\rho}\right)^{\alpha-1}(b-y)^{\rho-1} p(x) p(y)|H(x, y)| d x d y \\
\leq & \frac{1}{\Gamma^{2}(\alpha)} \int_{0}^{t} \int_{0}^{t}\left(\frac{(b-x)^{\rho}-(b-t)^{\rho}}{\rho}\right)^{\alpha-1}(b-x)^{\rho-1} \\
& \times\left(\frac{(b-y)^{\rho}-(b-t)^{\rho}}{\rho}\right)^{\alpha-1}(b-y)^{\rho-1} \\
& \times\left.\left.\left.\left. p(x) p(y)|x-y|\left|\int_{x}^{y}\right| f^{\prime}(u)\right|^{r} d u\right|^{r^{-1}}\left|\int_{x}^{y}\right| g^{\prime}(w)\right|^{s} d w\right|^{s^{-1}} d x d y .
\end{aligned}
$$

Applying again Hölder inequality to right-hand side of (14), we can write

$$
\begin{aligned}
& \frac{1}{\Gamma^{2}(\alpha)} \int_{0}^{t} \int_{0}^{t}\left(\frac{(b-x)^{\rho}-(b-t)^{\rho}}{\rho}\right)^{\alpha-1}(b-x)^{\rho-1} \\
& \times\left(\frac{(b-y)^{\rho}-(b-t)^{\rho}}{\rho}\right)^{\alpha-1}(b-y)^{\rho-1} p(x) p(y)|H(x, y)| d x d y \\
\leq & {\left[\frac{1}{\Gamma^{r}(\alpha)} \int_{0}^{t} \int_{0}^{t}\left(\frac{(b-x)^{\rho}-(b-t)^{\rho}}{\rho}\right)^{\alpha-1}(b-x)^{\rho-1}\right.} \\
& \left.\times\left.\left(\frac{(b-y)^{\rho}-(b-t)^{\rho}}{\rho}\right)^{\alpha-1}(b-y)^{\rho-1}|x-y| p(x) p(y)\left|\int_{x}^{y}\right| f^{\prime}(u)\right|^{r} d u \mid d x d y\right]^{r^{-1}} \\
& \times\left[\frac{1}{\Gamma^{s}(\alpha)} \int_{0}^{t} \int_{0}^{t}\left(\frac{(b-x)^{\rho}-(b-t)^{\rho}}{\rho}\right)^{\alpha-1}(b-x)^{\rho-1}\right. \\
& \left.\times\left.\left(\frac{(b-y)^{\rho}-(b-t)^{\rho}}{\rho}\right)^{\alpha-1}(b-y)^{\rho-1}|x-y| p(x) p(y)\left|\int_{x}^{y}\right| g^{\prime}(w)\right|^{s} d w \mid d x d y\right]^{s^{-1}} .
\end{aligned}
$$

Now, using the fact that

$$
\left.\left|\int_{x}^{y}\right| f^{\prime}(u)\right|^{r} d u\left|\leq\left\|f^{\prime}||_{r}^{r},\left.\quad\left|\int_{x}^{y}\right| g^{\prime}(w)\right|^{s} d w \mid \leq\right\| g^{\prime} \|_{s}^{s},\right.
$$

we obtain

$$
\begin{aligned}
& \frac{1}{\Gamma^{2}(\alpha)} \int_{0}^{t} \int_{0}^{t}\left(\frac{(b-x)^{\rho}-(b-t)^{\rho}}{\rho}\right)^{\alpha-1}(b-x)^{\rho-1} \\
& \times\left(\frac{(b-y)^{\rho}-(b-t)^{\rho}}{\rho}\right)^{\alpha-1}(b-y)^{\rho-1} p(x) p(y)|H(x, y)| d x d y \\
\leq & {\left[\frac{\left\|f^{\prime}\right\|_{r}^{r}}{\Gamma^{r}(\alpha)} \int_{0}^{t} \int_{0}^{t}\left(\frac{(b-x)^{\rho}-(b-t)^{\rho}}{\rho}\right)^{\alpha-1}(b-x)^{\rho-1}\right.}
\end{aligned}
$$




$$
\begin{aligned}
& \left.\times\left(\frac{(b-y)^{\rho}-(b-t)^{\rho}}{\rho}\right)^{\alpha-1}(b-y)^{\rho-1}|x-y| p(x) p(y) d x d y\right]^{r^{-1}} \\
& \times\left[\frac{\left\|g^{\prime}\right\|_{s}^{s}}{\Gamma^{s}(\alpha)} \int_{0}^{t} \int_{0}^{t}\left(\frac{(b-x)^{\rho}-(b-t)^{\rho}}{\rho}\right)^{\alpha-1}(b-x)^{\rho-1}\right. \\
& \left.\times\left(\frac{(b-y)^{\rho}-(b-t)^{\rho}}{\rho}\right)^{\alpha-1}(b-y)^{\rho-1}|x-y| p(x) p(y) d x d y\right]^{s^{-1}} .
\end{aligned}
$$

From (17), we get

$$
\begin{aligned}
& \frac{1}{\Gamma^{2}(\alpha)} \int_{0}^{t} \int_{0}^{t}\left(\frac{(b-x)^{\rho}-(b-t)^{\rho}}{\rho}\right)^{\alpha-1}(b-x)^{\rho-1} \\
& \times\left(\frac{(b-y)^{\rho}-(b-t)^{\rho}}{\rho}\right)^{\alpha-1}(b-y)^{\rho-1} p(x) p(y)|H(x, y)| d x d y \\
\leq & {\left[\frac{\left\|f^{\prime}\right\|_{r}\left\|g^{\prime}\right\|_{s}}{\Gamma^{2}(\alpha)} \int_{0}^{t} \int_{0}^{t}\left(\frac{(b-x)^{\rho}-(b-t)^{\rho}}{\rho}\right)^{\alpha-1}(b-x)^{\rho-1}\right.} \\
& \left.\times\left(\frac{(b-y)^{\rho}-(b-t)^{\rho}}{\rho}\right)^{\alpha-1}(b-y)^{\rho-1}|x-y| p(x) p(y) d x d y\right]^{r^{-1}} .
\end{aligned}
$$

Since $r^{-1}+s^{-1}=1$, then we have

$$
\begin{aligned}
& \frac{1}{\Gamma^{2}(\alpha)} \int_{0}^{t} \int_{0}^{t}\left(\frac{(b-x)^{\rho}-(b-t)^{\rho}}{\rho}\right)^{\alpha-1}(b-x)^{\rho-1} \\
& \times\left(\frac{(b-y)^{\rho}-(b-t)^{\rho}}{\rho}\right)^{\alpha-1}(b-y)^{\rho-1} p(x) p(y)|H(x, y)| d x d y \\
\leq & \frac{\left\|f^{\prime}\right\|_{r}\left\|g^{\prime}\right\|_{s}}{\Gamma^{2}(\alpha)}\left[\int_{0}^{t} \int_{0}^{t}\left(\frac{(b-x)^{\rho}-(b-t)^{\rho}}{\rho}\right)^{\alpha-1}(b-x)^{\rho-1}\right. \\
& \left.\times\left(\frac{(b-y)^{\rho}-(b-t)^{\rho}}{\rho}\right)^{\alpha-1}(b-y)^{\rho-1}|x-y| p(x) p(y) d x d y\right] .
\end{aligned}
$$

By the relations $(6)$ and $(19)$ and using the properties of the modulus, we get the first inequality in Theorem 17 .

Now we shall prove the second inequality of Theorem 17, we have

$$
0 \leq x \leq t, 0 \leq y \leq t
$$

Hence

$$
0 \leq|x-y| \leq t
$$


Therefore, we have

$$
\begin{aligned}
& \frac{1}{\Gamma^{2}(\alpha)} \int_{0}^{t} \int_{0}^{t}\left(\frac{(b-x)^{\rho}-(b-t)^{\rho}}{\rho}\right)^{\alpha-1}(b-x)^{\rho-1} \\
& \times\left(\frac{(b-y)^{\rho}-(b-t)^{\rho}}{\rho}\right)^{\alpha-1}(b-y)^{\rho-1} p(x) p(y)|H(x, y)| d x d y \\
\leq & \frac{\left\|f^{\prime}\right\|_{r}\left\|g^{\prime}\right\|_{s} t}{\Gamma^{2}(\alpha)}\left[\int_{0}^{t} \int_{0}^{t}\left(\frac{(b-x)^{\rho}-(b-t)^{\rho}}{\rho}\right)^{\alpha-1}(b-x)^{\rho-1}\right. \\
& \left.\times\left(\frac{(b-y)^{\rho}-(b-t)^{\rho}}{\rho}\right)^{\alpha-1}(b-y)^{\rho-1} p(x) p(y) d x d y\right] \\
= & \left\|f^{\prime}\right\|_{r}\left\|g^{\prime}\right\|_{s} t\left({ }_{0}^{b} \mathfrak{J}^{\alpha, \rho} p(t)\right)^{2} .
\end{aligned}
$$

Theorem 17 is thus proved.

Theorem 18. Let $p$ be a positive function on $[0, \infty[$ and let $f$ and $g$ be two differentiable functions on $\left[0, \infty\left[\right.\right.$. If $f^{\prime} \in L_{r}\left(\left[0, \infty[), g^{\prime} \in L_{s}\left(\left[0, \infty[), r^{-1}+s^{-1}=1\right.\right.\right.\right.$, then for all $t>0, \alpha, \beta, \rho>0$, we have

$$
\begin{aligned}
& \left.\mid \begin{array}{l}
b \mathfrak{J}_{0}^{\alpha, \rho} p(t) \\
{ }_{0}^{b} \mathfrak{J}^{\beta, \rho}
\end{array}\right] \text { fg }(t)+{ }_{0}^{b} \mathfrak{J}^{\beta, \rho} p(t){ }_{0}^{b} \mathfrak{J}^{\alpha, \rho} p f g(t) \\
& -{ }_{0}^{b} \mathfrak{J}^{\alpha, \rho} p f(t){ }_{0}^{b} \mathfrak{J}^{\beta, \rho} p g(t)-{ }_{0}^{b} \mathfrak{J}^{\beta, \rho} p f(t){ }_{0}^{b} \mathfrak{J}^{\alpha, \rho} p g(t) \mid \\
\leq & \frac{\left\|f^{\prime}\right\|_{r}\left\|g^{\prime}\right\|_{s}}{\Gamma(\alpha) \Gamma(\beta)} \int_{0}^{t} \int_{0}^{t}\left(\frac{(b-x)^{\rho}-(b-t)^{\rho}}{\rho}\right)^{\alpha-1}(b-x)^{\rho-1} \\
& \times\left(\frac{(b-y)^{\rho}-(b-t)^{\rho}}{\rho}\right)^{\beta-1}(b-y)^{\rho-1}|x-y| p(x) p(y) d x d y \\
\leq & \left\|f^{\prime}\right\|_{r}\left\|g^{\prime}\right\|_{s} t_{0}^{b} \mathfrak{J}^{\alpha, \rho} p(t){ }_{0}^{b} \mathfrak{J}^{\beta, \rho} p(t),
\end{aligned}
$$

where $H(x, y)$ are the same as given in 6 .

Proof. Using the identity (7), we can write

$$
\begin{aligned}
& \frac{1}{\Gamma(\alpha)} \frac{1}{\Gamma(\beta)} \int_{0}^{t} \int_{0}^{t}\left(\frac{(b-x)^{\rho}-(b-t)^{\rho}}{\rho}\right)^{\alpha-1}(b-x)^{\rho-1} \\
& \times\left(\frac{(b-y)^{\rho}-(b-t)^{\rho}}{\rho}\right)^{\alpha-1}(b-y)^{\rho-1} p(x) p(y) H(x, y) d x d y \\
= & { }_{0}^{b} \mathfrak{J}^{\alpha, \rho} p(t){ }_{0}^{b} \mathfrak{J}^{\beta, \rho} p f g(t)+{ }_{0}^{b} \mathfrak{J}^{\beta, \rho} p(t){ }_{0}^{b} \mathfrak{J}^{\alpha, \rho} p f g(t) \\
& -{ }_{0}^{b} \mathfrak{J}^{\alpha, \rho} p f(t){ }_{0}^{b} \mathfrak{J}^{\beta, \rho} p g(t)-{ }_{0}^{b} \mathfrak{J}^{\beta, \rho} p f(t){ }_{0}^{b} \mathfrak{J}^{\alpha, \rho} p g(t) .
\end{aligned}
$$

From the relation $[13)$, we can obtain the following estimation

$$
\frac{1}{\Gamma(\alpha)} \int_{0}^{t}\left(\frac{(b-x)^{\rho}-(b-t)^{\rho}}{\rho}\right)^{\alpha-1}(b-x)^{\rho-1} p(x)|H(x, y)| d x
$$




$$
\begin{aligned}
\leq & \frac{1}{\Gamma(\alpha)} \int_{0}^{t}\left(\frac{(b-x)^{\rho}-(b-t)^{\rho}}{\rho}\right)^{\alpha-1}(b-x)^{\rho-1}|x-y| p(x) \\
& \times\left.\left.\left.\left.\left|\int_{x}^{y}\right| f^{\prime}(u)\right|^{r} d u\right|^{r^{-1}}\left|\int_{x}^{y}\right| g^{\prime}(w)\right|^{s}\right|^{s^{-1}} d x .
\end{aligned}
$$

Therefore, we have

$$
\begin{aligned}
& \frac{1}{\Gamma(\alpha)} \frac{1}{\Gamma(\beta)} \int_{0}^{t} \int_{0}^{t}\left(\frac{(b-x)^{\rho}-(b-t)^{\rho}}{\rho}\right)^{\alpha-1}(b-x)^{\rho-1} \\
& \times\left(\frac{(b-y)^{\rho}-(b-t)^{\rho}}{\rho}\right)^{\beta-1}(b-y)^{\rho-1} p(x) p(y)|H(x, y)| d x d y \\
\leq & \frac{1}{\Gamma(\alpha) \Gamma(\beta)} \int_{0}^{t} \int_{0}^{t}\left(\frac{(b-x)^{\rho}-(b-t)^{\rho}}{\rho}\right)^{\alpha-1}(b-x)^{\rho-1} \\
& \times\left(\frac{(b-y)^{\rho}-(b-t)^{\rho}}{\rho}\right)^{\beta-1}(b-y)^{\rho-1}|x-y| p(x) p(y) \\
& \times\left.\left.\left.\left.\left|\int_{x}^{y}\right| f^{\prime}(u)\right|^{r} d u\right|^{r^{-1}}\left|\int_{x}^{y}\right| g^{\prime}(w)\right|^{s}\right|^{s^{-1}} d x d y .
\end{aligned}
$$

Applying Hölder inequality for double integral to the right-hand side of (24), yields

$$
\begin{aligned}
& \frac{1}{\Gamma(\alpha)} \frac{1}{\Gamma(\beta)} \int_{0}^{t} \int_{0}^{t}\left(\frac{(b-x)^{\rho}-(b-t)^{\rho}}{\rho}\right)^{\alpha-1}(b-x)^{\rho-1} \\
& \times\left(\frac{(b-y)^{\rho}-(b-t)^{\rho}}{\rho}\right)^{\beta-1}(b-y)^{\rho-1} p(x) p(y)|H(x, y)| d x d y \\
\leq & {\left[\frac{1}{\Gamma^{r}(\alpha)} \int_{0}^{t} \int_{0}^{t}\left(\frac{(b-x)^{\rho}-(b-t)^{\rho}}{\rho}\right)^{\alpha-1}(b-x)^{\rho-1}\right.} \\
& \times\left(\frac{(b-y)^{\rho}-(b-t)^{\rho}}{\rho}\right)^{\beta-1}(b-y)^{\rho-1}|x-y| p(x) p(y) \\
& \left.\times\left.\left|\int_{x}^{y}\right| f^{\prime}(u)\right|^{r} d u \mid d x d y\right]^{r^{-1}} \\
& \times\left[\frac{1}{\Gamma^{s}(\beta)} \int_{0}^{t} \int_{0}^{t}\left(\frac{(b-x)^{\rho}-(b-t)^{\rho}}{\rho}\right)^{\alpha-1}(b-x)^{\rho-1}\right. \\
& \times\left(\frac{(b-y)^{\rho}-(b-t)^{\rho}}{\rho}\right)^{\beta-1}(b-y)^{\rho-1}|x-y| p(x) p(y) \\
& \left.\times\left.\left|\int_{x}^{y}\right| g^{\prime}(w)\right|^{s} d w \mid d x d y\right]^{s^{-1}} .
\end{aligned}
$$


By 16 and 25), we get

$$
\begin{aligned}
& \frac{1}{\Gamma(\alpha)} \frac{1}{\Gamma(\beta)} \int_{0}^{t} \int_{0}^{t}\left(\frac{(b-x)^{\rho}-(b-t)^{\rho}}{\rho}\right)^{\alpha-1}(b-x)^{\rho-1} \\
& \times\left(\frac{(b-y)^{\rho}-(b-t)^{\rho}}{\rho}\right)^{\beta-1}(b-y)^{\rho-1} p(x) p(y)|H(x, y)| d x d y \\
\leq & \frac{\left\|f^{\prime}\right\|_{r}\left\|g^{\prime}\right\|_{s}}{\Gamma(\alpha) \Gamma(\beta)} \int_{0}^{t} \int_{0}^{t}\left(\frac{(b-x)^{\rho}-(b-t)^{\rho}}{\rho}\right)^{\alpha-1}(b-x)^{\rho-1} \\
& \times\left(\frac{(b-y)^{\rho}-(b-t)^{\rho}}{\rho}\right)^{\beta-1}(b-y)^{\rho-1}|x-y| p(x) p(y) d x d y .
\end{aligned}
$$

Using $(22)$ and $(26)$ and the properties of modulus, we get the first inequality in (21).

\section{REMARKS}

Now, let us briefly consider some special cases of the main results. In Theorem 17 and Theorem 18, if we choose $\rho=1$ and make use of the relationship (3), then the main results are reduced to Theorem 2 and Theorem 3 obtained by Dahmani et al. [7].

\section{REFERENCES}

[1] Abdeljawad, T., Fractional operators with boundary points dependent kernels and integration by parts, Discrete and Continuous Dynamical Systems Series S, 13(3) (2020), 351-375.

[2] Baleanu, D., Purohit, S.D., Chebyshev Type Integral Inequalities Involving the Fractional Hypergeometric Operators, Abstract and Applied Analysis, 2014 (2014), Article ID 609160, $1-10$.

[3] Baleanu, D., Purohit, S.D., Prajapati, J.C., Integral inequalities involving generalized Erdélyi-Kober fractional integral operators, Open Mathematics, 14 (2016), 89-99.

[4] Chebyshev, P.L., Sur les expressions approximatives des integrales definies par les autres prises entre les mêmes limites, Proc. Math. Soc. Charkov, 2 (1882), 93-98.

[5] Curiel, L., Galué, L., A generalization of the integral operators involving the Gauss hypergeometric function, Revista Técnica de la Facultad de Ingeniería Universidad del Zulia, 19(1) (1996), 17-22.

[6] Çelik, B., Set, E., Akdemir, A.O., Mixed Conformable Fractional Grüss-Type Inequalities, 2nd International Conference on Life and Engineering Sciences (ICOLES 2019), Book of Abstracts, Istanbul, Turkey, 2019.

[7] Dahmani, Z., Mechouar, O. and Brahami, S., Certain inequalities related to the Chebyshev's functional involving a Riemann-Liouville operator, Bull. Math. Anal. Appl., 3(4) (2011), $38-44$.

[8] Ekinci, A., Özdemir, M.E., Some new integral Inequalities via Riemann Liouville integral operators, Applied and Computational Mathematics, 18(3) (2019), 288-295.

[9] Farid, G., Existence of an integral operator and its consequences in fractional and conformable integrals, Open Journal of Mathematical Sciences, 3 (2019), 210-216.

[10] Gürbüz, M., Özdemir, M.E., On Some Inequalities for Product of Different Kinds of Convex Functions, Turkish Journal of Science, 5(1) (2020), 23-27. 
[11] Kilbas, A.A., Srivastava, H.M., Trujillo, J.J., Theory and Applications of Fractional Differential Equations, vol. 204 of North-Holland Mathematics Studies, Elsevier, Amsterdam, The Netherlands, 2006.

[12] Kiryakova, V., Generalized Fractional Calculus and Applications, vol. 301 of Pitman Research Notes in Mathematics Series, Longman Scientific \& Technical, Harlow, UK, 1994.

[13] Ntouyas, S.K., Purohit, S.D., Tariboon, J., Certain Chebyshev Type Integral Inequalities Involving Hadamard's Fractional Operators, Abstract and Applied Analysis, 2014 (2014), Article ID 249091, 1-7.

[14] Özdemir, M.E., Set, E., Akdemir, A.O., Sarıkaya, M.Z., Some new Chebyshev type inequalities for functions whose derivatives belongs to $L_{p}$ spaces, Afrika Matematika, 26 (2015), $1609-1619$.

[15] Rahman, G., Baleanu, D., Qurashi, M.A., Purohit, S.D., Mubeen, S., Arshad, M., The extended Mittag-Leffler function via fractional calculus, J. Nonlinear Sci. Appl., 10 (2017), $4244-4253$.

[16] Sarıkaya, M.Z., Alp, N., On Hermite-Hadamard-Fejer type integral inequalities for generalized convex functions via local fractional integrals, Open Journal of Mathematical Sciences, 3(1) (2019), 273-284.

[17] Set, E., Özdemir, M.E., Demirbaş, S., Chebyshev type inequalities involving extended generalized fractional integral operators, AIMS Mathematics, 5(4) (2020), 3573-3583.

[18] Set, E., New Inequalities of Chebyshev Type for Mixed Conformable Fractional Integral Operators, 2nd International Conference on Life and Engineering Sciences (ICOLES 2019), Book of Abstracts, Istanbul, Turkey, 2019.

[19] Srivastava, H.M., Choi, J., Zeta and $q$-Zeta Functions and Associated Series and Integrals, Elsevier Science Publishers, Amsterdam, London and New York, 2012. 